\section{Applied Beams, LLC}

Applied Beams LLC is dedicated to extending the life and enhancing the performance of the ever growing installed base of FIB and SEM systems around the world. We are a leading supplier of FIB and SEM consumables, micro-machining services, and system upgrades. With 30+ years of experience with ion and electron beam systems design, manufacture and applications we provide a unique ability to meet customer needs with these powerful technologies.

We serve the semiconductor, materials analysis and research communities, and proudly support the US Government with our unique ability to develop and deliver custom FIB and SEM systems.

- FIB and SEM Consumables - Our high quality, affordable extractors, suppressors, liquid metal ion sources, Schottky electron sources, and aperture strips are direct replacements for original components, keeping your FIB, SEM and dual column FIB-SEM systems operating at optimum performance and on budget.

- HyperFIB upgrade - Our high-current HyperFIB system is the only plasma FIB upgrade on the market for legacy FIB tools, increasing available current for micromachining, crosssectioning and material deposition from just $20 \mathrm{nA}$ to over $7 \mathrm{uA}$, an improvement of 350X!

- Caesium control system upgrade - Extend the performance and life of your FEI/Philips XL-series or Camscan SEM with our Caesium ${ }^{\text {tw }}$ series control system upgrade. Derived from CamScan Helios ${ }^{\mathrm{st}}$ software, Caesium runs on the Microsoft Windows 10 operating system with new features, new capabilities, and brings your XL-series or Camscan SEM into the 21st century.

Advanced features include:

- Multiple image windows, up to 20 image panes per window. Display multiple live images in real-time

- Support for multiple USB cameras allows in-chamber viewing on the Caesium screen, with integrated camera controls

- Image processing: contrast, brightness and gamma adjustments, filters, edge detection and more.

- Image calibration and measurements

- Image annotation and drawing tools - saved with or "burned into" the image.

- Drag and drop images into Caesium from Windows Explorer and other applications

- Automate operations using a simple text scripts in the Python language, example scripts available to get you started.

- Control your SEM remotely using Windows Remote Desktop feature

- Image notes and tool properties display in Windows Explorer

- Store and automatically recall individual user settings

- Image reflection for optical microscope camera displays

- Save image in tiff, bmp, jpeg, gif or png formats. Your pre-existing images of course are all imported to your new control PC.

The Caesium control system upgrade is available as a complete PC+software package shipped direct from our factory. We provide Dell computers for the best in quality and worldwide service and support. Caesium is designed for customer installation with email and phone support as needed, or we can handle installation for you - just let us know which you prefer.

- Micromachining and analytical services - Our micromachining services utilize our unique HyperFIB systems. With up to $7 \mathrm{uA}$ of beam current this tool is perfect for preparing cross-sections of advanced semiconductor devices and delayering all or a portion of a semiconductor device.

Buy with confidence. Our customers include Fortune 500 companies, top research institutions and government laboratories around the world.

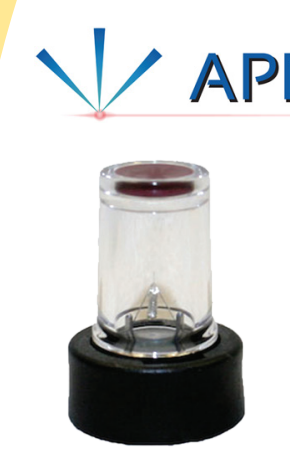

Liquid Metal Ion Sources

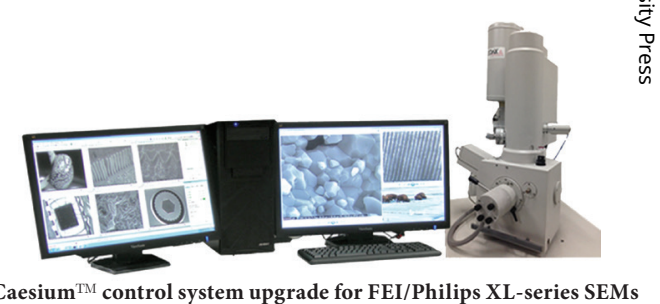

Caesium $^{\mathrm{TM}}$ control system upgrade for FEI/Philips XL-series SEMs

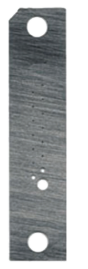

FIB and SEM Aperture Strips

\section{How to find us}

\author{
14855 S.W. Murray Scholls Drive, \\ Beaverton, Oregon 97007 USA \\ Tel: +1 (503) 608-7237 \\ Fax: +1 (503) 214-8057 \\ Email: info@appliedbeams.com \\ www.appliedbeams.com
}

